American Journal of Applied Sciences 5 (9): 1107-1109, 2008

ISSN 1546-9239

(C) 2008 Science Publications

\title{
Strongly Real Elements in the Linear Groups $\operatorname{PSL}_{\mathbf{n}}(\mathbf{q})$
}

\author{
Ibrahim A.I. Suleiman \\ Department of Mathematics and Statistics, Mu'tah University, \\ P.O.Box (7), 61710 Al-Karak, Jordan
}

\begin{abstract}
We investigated which elements in some projective special linear and unitary groups are strongly real. In particular we showed every real element in $\mathrm{PSL}_{2}(\mathrm{q})$ strongly real. We write a full table for real classes which are not strongly real in the unitary groups as well as a table for the non real classes in the unitary groups.
\end{abstract}

Key words: Real elements, linear groups

\section{INTRODUCTION}

An element $\mathrm{g}$ in a group $\mathrm{G}$ is called real if $\mathrm{g}$ is conjugate to $\mathrm{g}^{-1}$ and is called strongly real if it is the product of two involutions. Similarly the conjugacy class $\mathrm{g}^{\mathrm{G}}$ is called real or strongly real if $\mathrm{g}$ is. Clearly every strongly real element is real, but the converse is not true. For example, the quaternion group $\mathrm{Q}_{8}$ has the property that all its elements are real, but its only involution is central, so cannot conjugate an element of order 4 to its inverse. In a recent study ${ }^{[2]}$, Tiep and Zalesski determine which finite simple groups have the property that every element is real. A much more difficult question, raised in problem 14.82 of the Kourovka notebook ${ }^{[6]}$, is which finite simple groups have the property that every element is strongly real. This problem is still open in general (but see ${ }^{[3,4]}$, for some cases, and related questions). In a preprint ${ }^{[7]}$, it has been determined exactly which elements in the sporadic and the alternating groups are strongly real. In the alternating groups every real element is strongly real, but this is not true in all sporadic groups. The alternating and the sporadic groups in which every element is strongly real are An for $(\mathrm{n}=5,6,10,14)$, and $\mathrm{J}_{1}$ and $\mathrm{J}_{2}$. It is known ${ }^{[8,9]}$ ) that all real elements in the general linear group $\mathrm{GL}_{\mathrm{n}}(\mathrm{q})$ are strongly real. However, this is not true in the special linear groups $\mathrm{SL}_{\mathrm{n}}$ (q) for example, if $\mathrm{n}=2$, then there is only one involution in $\mathrm{SL}_{2}(\mathrm{q})$, so only the central elements of $\mathrm{SL}_{2}$ (q) are strongly real. On the other hand, we prove that all real elements in $\mathrm{PSL}_{2}$ (q) are strongly real. Indeed, we conjecture that all real elements in $\mathrm{PSL}_{\mathrm{n}}$ (q) are strongly real, for arbitrary n. Moreover, we completely classify the strongly real elements in many unitary groups as far as they are $\mathrm{in}^{[1]}$. We used $\mathrm{GAP}^{[5]}$ to find all elements in all these groups which are real but not strongly real.

\section{GENERAL LINEAR GROUPS}

The general linear group $\mathrm{GL}_{\mathrm{n}}$ (q) consists of all $\mathrm{n} \times \mathrm{n}$ matrices with entries in $\mathrm{F}_{\mathrm{q}}$. Equivalently it is the group of all linear automorphisms of an n-dimensional vector space over $\mathrm{F}_{\mathrm{q}}$. The special linear group $\mathrm{SL}_{\mathrm{n}}(\mathrm{q})$ is the subgroup of all matrices of determinant 1 . On factoring $\mathrm{GL}_{\mathrm{n}}(\mathrm{q})$ and $\mathrm{SL}_{\mathrm{n}}(\mathrm{q})$ by the scalar matrices we get what is known as the projective general linear group $\mathrm{PGL}_{\mathrm{n}}$ (q) and projective special linear group $\mathrm{PSL}_{n}$ (q). The orders of these groups are given by the following formulae:

$$
\begin{aligned}
& \left|\mathrm{GL}_{\mathrm{n}}(\mathrm{q})\right|=(\mathrm{q}-1) \mathrm{N},\left|\mathrm{SL}_{\mathrm{n}}(\mathrm{q})\right| \\
& =\left|\mathrm{PGL}_{\mathrm{n}}(\mathrm{q})\right|=\mathrm{N}
\end{aligned}
$$

and

$$
\left|P L_{n}(q)\right|=\left|L_{n}(q)\right|=\frac{N}{d}
$$

where,

$$
\mathrm{N}=\mathrm{q}^{\mathrm{n}(\mathrm{n}-1) / 2}\left(\mathrm{q}^{\mathrm{n}}-1\right) \ldots\left(\mathrm{q}^{2}-1\right)
$$

and $\mathrm{d}$ is the greatest common divisor of $\mathrm{q}-1$ and $\mathrm{n}$.

Projective special linear groups In (q): We know from $^{[2]}$, that the only groups $L_{n}(q)$ in which all elements are real are the groups $L_{n}(q)$ with either $q=2^{k}$ or $\mathrm{q} \equiv 1 \bmod 4$. Here we show that in fact all

Current Address: Ibrahim A.I. Suleiman, Department of Mathematical and Physical Sciences, University of Nizwa, Birkat ALMouz, P.O. Box 33, 616 Nizwa, Sultanate of Oman 
elements in these groups are strongly real. We also determine which elements are strongly real in $L_{n}(q)$ for $\mathrm{q} \equiv 3 \bmod 4$.

Proposition 1: In this case the elements have order dividing $\mathrm{L}_{2}\left(2^{\mathrm{k}}\right)$, where $\mathrm{k}>1$, are strongly real.

Proof: The elements of $\mathrm{L}_{2}\left(2^{\mathrm{k}}\right)$ have order dividing 2 , or $2^{\mathrm{k}}-1$, or $2^{\mathrm{k}}+1$. Indeed the normalizers of the cyclic groups of orders $2,2^{\mathrm{k}}-1$, and $2^{\mathrm{k}}+1$ are $\mathrm{C}_{2}^{\mathrm{k}}, \mathrm{D}_{2\left(2{ }^{\mathrm{k}}-1\right)}$ and $D_{2(2+1)}{ }^{k}$ respectively. Since $C_{2}^{k}>\left(C_{2} \times C_{2}\right)=D_{4}$, the result follows.

Proposition 2: All elements in $\mathrm{L}_{\mathrm{n}}(\mathrm{q})$, where $\mathrm{q} \equiv 1 \mathrm{mod}$ 4 , are strongly real.

Proof: In this case the elements of order divide either $\mathrm{p}$ (where $\mathrm{p}$ is the prime dividing $\mathrm{q}$ ), or $(\mathrm{q}-1) / 2$ or $(\mathrm{q}+1) / 2$. In the last two cases, the normalizers of the corresponding cyclic groups are now $\mathrm{D}_{\mathrm{q}-1}$ and $\mathrm{D}_{\mathrm{q}+1}$, so all these classes are strongly real. In the first case, the normalizer of the cyclic group is contained in the Borel subgroup $\mathrm{C}_{\mathrm{p}}^{\mathrm{k}}: \mathrm{C}_{(\mathrm{q}-1)}$, where $\mathrm{q}=\mathrm{p}^{\mathrm{k}}$. Because $\mathrm{q} \equiv 1$ $\bmod 4$, this subgroup contains $\mathrm{C}_{\mathrm{p}}^{\mathrm{k}}: \mathrm{C}_{2}$, in which the $\mathrm{C}_{2}$ conjugates all elements in the $\mathrm{C}_{\mathrm{p}}^{\mathrm{k}}$ to their inverses. Therefore all these elements are strongly real also.

Proposition 3: All real elements in $L_{n}(q)$, where $q \equiv 3$ $\bmod 4$, are strongly real.

Proof: In this case the elements of order $p$, (where $p$ is the prime dividing $\mathrm{q}$ ) are not real. All other elements have order dividing $(q-1) / 2$ or $(q+1) / 2$. The normalizers of the corresponding cyclic groups are again $\mathrm{D}_{\mathrm{q}-1}$ and $\mathrm{D}_{\mathrm{q}+1}$, so all these classes are strongly real.

Unitary Groups: The projective general unitary group $\mathrm{PGU}_{n}(\mathrm{q})$ is the subgroup of all elements of GL $\left(\mathrm{q}^{2}\right)$ that fix the corresponding non-singular conjugatesymmetric sesquilinear form. The determinant of a unitary matrix is necessarily a $(q+1)^{\text {st }}$ of unity. The projective general unitary group $\operatorname{PSU}_{\mathrm{n}}(\mathrm{q})$ is the subgroup of unitary matrices of determinant 1 . On factoring $\mathrm{PGU}_{\mathrm{n}}(\mathrm{q})$ and $\mathrm{PSU}_{\mathrm{n}}(\mathrm{q})$ by the scalar matrices we get what is known as the special unitary group $\mathrm{PGU}_{\mathrm{n}}(\mathrm{q})$ and the projective special unitary group $\mathrm{PSU}_{\mathrm{n}}$ (q). The orders of these groups are given by the following formulae:

$$
\begin{aligned}
& \left|G U_{n}(q)\right|=(q+1) N,\left|S U_{n}(q)\right| \\
& =\left|P G U_{n}(q)\right|=N
\end{aligned}
$$

and

$$
\left|\operatorname{PSU}_{n}(q)\right|=\left|U_{n}(q)\right|=\frac{N}{d}
$$

where,

$$
N=q^{n(n-1) / 2}\left(q^{n}-(-1)^{n}\right) \ldots . .\left(q^{3}+1\right)\left(q^{2}-1\right)
$$

and $\mathrm{d}$ is the greatest common divisor of $\mathrm{q}+1$ and $\mathrm{n}$.

In our case for the unitary groups, we can check using the character table whether each element is a product of two involutions. For element $\mathrm{z}$ in a conjugacy class $\mathrm{Z}$ is a product of elements $\mathrm{x}$ and $\mathrm{y}$ in classes $\mathrm{X}$ and $\mathrm{Y}$ respectively if and only if the structure constant:

$\xi(\mathrm{X}, \mathrm{Y}, \mathrm{Z})=\frac{|\mathrm{G}|}{\left|\mathrm{C}_{\mathrm{G}}(\mathrm{x})\right|\left|\mathrm{C}_{\mathrm{G}}(\mathrm{y})\right|_{\chi}} \sum_{\chi} \frac{\chi(\mathrm{x}) \chi(\mathrm{y})^{--} \chi(\mathrm{z})}{\chi(1)}$

is non zero. Indeed $\xi(\mathrm{X}, \mathrm{Y}, \mathrm{Z})$ is well-known to be equal to the number of ways that $\mathrm{z}$ can be expressed as a product of $x y$ with $x$ in $X$ and $y$ in $Y$. Thus to determine if $\mathrm{z}$ is a product of two involutions, we only need to add up the structure constants $\xi(\mathrm{X}, \mathrm{Y}, \mathrm{Z})$ over classes $\mathrm{X}, \mathrm{Y}$ of involutions, and we see whether the resulting integer is positive or zero. In the study ${ }^{[2]}$, Tiep and Zalesski determine that unitary groups have nonreal elements. In this section we completely classify the strongly real elements in all unitary groups as far as they are $i^{[1]}$. Table 1 gives us all elements which are real but not strongly real. For completeness, we also list all the non real elements in the unitary groups. Table 2 is the complete list for all these non real elements.

Table 1: Real classes which are not strongly real in unitary groups

U3 (3) 3A, 6A.

U4 (2) all real elements are strongly real.

U3 (4) $\quad 4 \mathrm{~A}$

U3 (5) 5A, 10A.

U4 (3) 3A, 6A, 8A, 12A.

U3 (9) 3A, 6A.

U3 (11) 11A, 22A.

U6 (2) $8 \mathrm{~A}$

U5 (2) all real elements are strongly real.

U3 (7) all real elements are strongly real.

U3 (8) all real elements are strongly real. 
Table 2: Non- Real classes in unitary groups

U3 (3) $4 \mathrm{AB}, 7 \mathrm{AB}, 8 \mathrm{AB}, 12 \mathrm{AB}$

$\mathrm{U} 4$ (2) $3 \mathrm{AB}, 6 \mathrm{AB}, 6 \mathrm{CD}, 9 \mathrm{AB}, 12 \mathrm{AB}$

U3 (4) 5ABCD, 10ABCD, 13ABCD, 15ABCD

$\mathrm{U} 3$ (5) $7 \mathrm{AB}, 8 \mathrm{AB}$

U4 (3) 7AB, 9ABCD

U3 (9) 5ABCD, 10ABCD, 10G- N, 20F . . 80-P

U3 (11) 4AB, 8AB, 10BCD, 12AB, 20ABCD, 37A -M, 40A -H, 4AB

$\mathrm{U} 6(2) \quad 6 \mathrm{AB}, 9 \mathrm{AB}, 11 \mathrm{AB}, 12 \mathrm{ABDE}, 18 \mathrm{AB}$

\section{ACKNOWLEDGEMENTS}

This work has been completed through a sabbatical year at the University of Nizwa -Sultanate of Oman. This year was offered by Mutah University - Jordan.

\section{REFERENCES}

1. Conway, J.H., R.T. Curtis, S.P. Norton, R.A. Parker and R.A. Wilson, 1985. An ATLAS of Finite Groups, Clarendon Press, Oxford.

2. Pham Tiep, A.E. Zalesski, 2005. Real conjugacy classes in algebraic groups and finite groups of Lie type, J. Group Theory, 8: 291-315.

3. Gow, R., 1985. Real representations of the finite orthogonal and Symplectic groups of odd characteristic. J. Algebra, 96: 249-274.
4. Gow, R., 1981. Products of two involutions in classical groups of characteristic 2, J. Algebra 71: 583-591.

5. Schonert, M. et al., 1994. GAP 3.4 Manual (Groups, algorithms, and Programming), RWTH Aachen.

6. Khukhro, E. and V. Mazurov, 2002. The Kourovka Notebook, 14th Edn., Novosibirsk

7. Ibrahim, A.I., 2008. Suleiman, Strongly real elements in the sporadic and the alternating groups, (In Press).

8. Wonenburger, M.J., 1966. Transformations which are the products of two involutions, J. Math.16: 327-338.

9. Djokovic, D., 1967. Product of two involutions, Arch. Math. (Basel) 18: 582-584. 